\title{
Processing System ANd Algorithms for the TANDEM-X Mission
}

\author{
H. Breit, T. Fritz, M. Eineder, R. Bamler, M. Lachaise, R. Brcic, N. Adam, and N. Yague-Martinez
}

Remote Sensing Technology Institute, German Aerospace Center DLR

\begin{abstract}
In 2009, the German radar satellite TerraSAR-X will be supplemented with the TanDEM-X satellite to form the first bi-static single pass interferometer in space. TanDEM-X will fly close to TerraSAR-X in a controlled helix configuration for 3 years to jointly acquire interferometric SAR data in bistatic mode. The primary TanDEM-X mission goal is to generate a global Digital Elevation Model (DEM) with a relative point-to-point height accuracy of 2 meters for moderate terrain at $12 \mathrm{~m}$ posting [1]. This paper outlines the SAR data workflow from quality check screening through to bistatic focusing and interferometric processing to raw DEM generation.
\end{abstract}

Index Terms - TanDEM-X, Bistatic SAR, Digital Elevation Model (DEM), SAR Interferometry

\section{INTRODUCTION}

First interferometric processing results from TerraSAR-X repeat-pass acquisitions impressively demonstrate the details visible in DEMs with 6 to $10 \mathrm{~m}$ spacing and meter level accuracy [1]. Major obstacles to accurate DEM generation, namely atmospheric phase errors and temporal decorrelation will be mitigated by the quasi simultaneous interferometric imaging of the TanDEM-X mission so that homogenous DEMs are expected all over the entire globe. Since TerraSAR-X was not originally designed as a global mapping system, careful planning is required in order to achieve the planned 2-4 global coverages within the 3 year mission duration. The TanDEM-X project is partly funded by the German Federal Ministry for Economics and Technology (Förderkennzeichen 50 EE 0601).

\section{THE TANDEM-X MISSION PHASES}

The TanDEM-X mission is characterized by different phases which are clearly distinguished by the formation flight parameters and the radar imaging modes. The geometric configuration as well as the capability to perform joint SAR operations in different cooperative modes (bistatic, alternating bistatic, monostatic or experimental ones) adds an additional dimension to the diversity of possible acquisition modes and thus poses different requirements and challenges to the ground operations and processing systems for each phase.
According to the current TanDEM-X mission operations concept, in the first weeks after launch, the TDX-1 satellite will be steered into an orbit separated by approximately $20 \mathrm{~km}$ along track from the TSX-1 satellite. This configuration will be maintained for 3 months. In this "pursuit monostatic" commissioning phase where the two SAR instruments do not interfere, the geometric, radiometric and pointing calibration of the TDX-1 satellite as an independently operating instrument system will be performed. In this phase, the operational processing system of the TerraSAR-X mission with the TerraSAR-X MultiMode SAR Processor (TMSP) is the main workhorse. The small 3 second time lag between satellites enables cross calibration and first joint interferometric tests with small temporal decorrelation. The monostatic operation of both satellites with this small time lag is also a nominal "cooperative mode" which is processed by the Integrated TanDEM-X Processor (ITP). It is also foreseen as a fallback mode for operational DEM generation - albeit with reduced performance.

After the TDX instrument is commissioned to support data acquisition for standalone SAR product generation, the close formation helix configuration will be safely built up for cooperative operation tests with both instruments. This bistatic commissioning phase is required to adjust and verify among others the phase synchronization accuracy, calibration of the bistatic signal paths, error models and the ground segment operations including formation control, to mention a few. All processing of joint TanDEM-X acquisitions is done by the operational ITP.

Starting the close formation with a wider across track component and correspondingly larger effective baselines, the resulting bistatic test data takes will also be used to gather DEM data with increased height sensitivity to test the acquisition geometries anticipated in later mission phases. After the bistatic commissioning phase, there will be at least two complete coverages of the global land surface for operational DEM data acquisition with each lasting approximately one year. They will have different heights of ambiguity to allow multi-baseline phase unwrapping. The first year of operation starts in the closest formation with lower height accuracy but less sensitivity to phase unwrapping errors. This yields data suitable for the production of an intermediate global DEM. The second coverage with its larger baselines will add the height 
sensitivity necessary to achieve the required final DEM accuracy but the phase unwrapping must be supported by the first global coverage.

Finally, difficult terrain will be covered again with different acquisition geometries, i.e. different look direction and/or incidence angles. To achieve the required accuracy and for the sake of homogenous data quality, the acquisitions from different mission phases will be fused into the final global DEM. Operational DEM data acquisitions are complemented by experimental acquisitions in different cooperative modes, e.g. alternating bistatic mode.

\section{TANDEM-X SAR DATA FLOW}

On top of the ongoing operational TerraSAR-X data imaging mission which will be distributed between the two satellites, the TanDEM-X mission will gather DEM data which corresponds to 2 x 400 TerraSAR-X stripmap products per day. Besides the high data volume and throughput requirements, the operational processing chain has to meet the challenges of combining acquisitions from different mission phases which are downlinked in segments over widely distributed remote ground stations and yet provide a fast feedback on interferometric data quality for (re-) acquisition planning well ahead of flight formation changes.

The quality assessment and fast feedback is achieved by establishing a data quality screening process at the receiving stations. This ITP-Screener provides small result files which are sent quickly to the central processing facility, collected and combined for each joint DEM acquisition. The following parameter evaluation step by the central ITP installation assesses e.g. the quality of the synchronization pulse analysis, the raw data statistics and the Doppler centroid estimation to indicate problematic data takes. This screening allows the instrument data to be shipped, combined and processed with a certain delay without loosing track of acquisition quality.

The processing of DEM acquisitions follows a consistent systematic and data driven approach. Upon availability of the complete raw data of one data take and the required auxiliary data, such as a precise baseline product, the ITP starts processing with a precise screening step for consistent SAR focusing and interferometric processing parameter determination of the two raw data sets. A framing into independently processed scenes is then applied to generate, in parallel, several focused and co-registered single-look slant-range complex (CoSSC) products of about $55 \mathrm{~km}$ in length which are corrected for all timing and oscillator drifts. These CoSSCs are stored for future use in multibaseline processing but are also directly processed to interferograms and the so called Raw DEMs. These are archived for later access by the mosaicking and calibration processor. Experimental products are processed with the same chain to coregistered images (CoSSCs) as user products, leaving out the Raw DEM generation step.

The processing concept thus follows a unified concept for the generation of the global DEM, customized DEMs and most of the experimental products.

\section{BISTATIC PROCESSING CHALLENGES}

Compared to the monostatic case, the essential challenges in processing SAR data acquired in a bistatic configuration are the imaging geometry and independently drifting oscillators of the two instruments [3].

\subsection{Determination of the equivalent velocity}

In the case of TanDEM-X which is characterized by its moderate bistatic acquisition geometry the shape of the true range histories deviate only slightly from hyperbolas [4]. For hyperbolic range histories an analytical formulation of the 2-D SAR transfer function exists. Standard SAR processors such as chirp scaling, typically implement focusing algorithms, which approximate the analytical transfer function. This makes it possible to reuse the chirp scaling based TerraSAR-X processor with only a few modifications. As shown in [4] highly accurate bistatic focusing is achieved by an appropriate approximation of the true range histories $R(t)$ by hyperbolic ones:

$$
R\left(t ; R_{0}\right) \approx \widetilde{R}\left(t ; R_{0}\right)=\sqrt{R_{0}^{2}+\widetilde{V}^{2} t^{2}}
$$

The so called equivalent velocity $\widetilde{V}$ has to be adjusted such that the monostatic hyperbola optimally approximates the true bistatic range history. For this, target ranges with respect to the transmitting and receiving satellite are numerically determined as functions of azimuth time by means of a series of orbit interpolations and geolocations. A sufficiently precise match of the shapes is obtained by adjusting $\widetilde{V}$ to the second derivative of the true range, $\widetilde{V}^{2}=R_{0} \ddot{R}(0)$. This adjustment is performed prior to focusing at grid-points with an $\sim 300$ m posting which cover the entire scene and take the local terrain height into account.

\subsection{Sync-Pulse Processing}

The more demanding challenge is caused by the independently drifting oscillators of both instruments. Thus, the SAR raw data of the passive bistatic channel is affected by sampling and time annotation distortions and strong phase modulations [5]. A thorough compensation is required in order to achieve the specified DEM quality. An indispensable prerequisite is the precise knowledge of the relative phase difference of both oscillators at any time during a bistatic acquisition. Therefore, both instruments continuously, at a rate of about $10 \mathrm{~Hz}$, exchange dedicated sync-pulses which are modulated to X-band and transmitted by one satellite, received, demodulated, and interspersed 
into the SAR raw data stream by the other satellite and vice versa [3], [5].

Sync-pulse processing is performed at the TanDEM-X receiving stations immediately after SAR data downlink. The interspersed chirp-type pulses are correlated by a nominal reference chirp and the results, phase and position of correlation peaks are transferred to the central processing facility. After evaluation, coarse frequency offset estimates are considered in the echo-window commanding of the passive channel of future acquisitions while highly precise estimates of phase deviations are directly used for correction of SAR raw data. Due to the fact that in worst case situations pixel size distortions of only a few $\mu$-meters in range would add up to meters after a few seconds, a line by line range shift correction is incorporated in the range processing step of the SAR processor.

\section{PHASE UNWRAPPING}

\subsection{Single baseline phase unwrapping}

Phase unwrapping is the most critical step in DEM reconstruction. A good implementation based on the minimum cost flow method (MCF) is available from the SRTM mission which processes single interferograms (single baseline phase unwrapping) [6]. This method is well suited for an estimated 50\% of all TanDEM-X scenes, but for large baselines and for difficult terrain this algorithm is not expected to be sufficient and new methods based on multi-baseline or even multi-incidence angle techniques must be applied [7], [8]. Algorithmic performance tests have been performed using simulated interferograms obtained from DEMs generated from operational TerraSAR-X stripmap products. DEM sections are replicated to allow a free selection of different geometric layouts.

\subsection{Dual baseline phase unwrapping}

The data acquired from the second coverage on will be jointly processed with the data from the first coverage to meet the DEM height error requirements. Since the two available interferograms are taken from the same viewing geometry, phase unwrapping can be performed in slant range geometry and thus needs less processing time. Several possibilities exist to achieve dual baseline phase unwrapping. As a first option, the differential interferogram between the two passes can be used. Secondly, both interferograms can be unwrapped separately and the unwrapped phases can be compared. Regions deviating by integer multiples of the height of ambiguity are corrected with respect to each other by region growing algorithms for example. The third way is to use a simplified version of an existing maximum likelihood estimator (MLE) published in [7] to slant range geometry. However since only two interferograms are available, the result of the MLE is very noisy. To circumvent this problem, some a priori knowledge is needed, e.g. from a lower resolution reference DEM
(SRTM) or the delta-k phase from split bandwidth processing [9], [10], [11] (see Figure 1). Since the delta-k phase estimation is incorporated in the processing chain, this is the currently implemented solution.
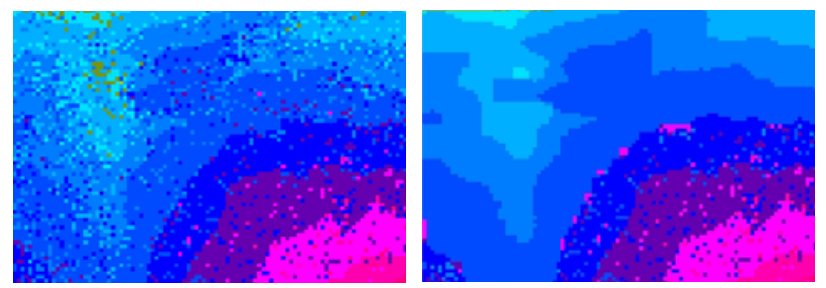

Figure 1: Left hand side, a zoom into the unwrapped phase with using MLE; right hand side, unwrapped phase using a prior knowledge which smoothes the terrain

A fourth option which is being studied for further optimization is to perform the maximum likelihood estimation on gradients. Processing time is reduced since the range of the search is only two to four cycles, see Figure 2. The unwrapped gradients can then be used MCF phase unwrapping to reduce the number of residues.

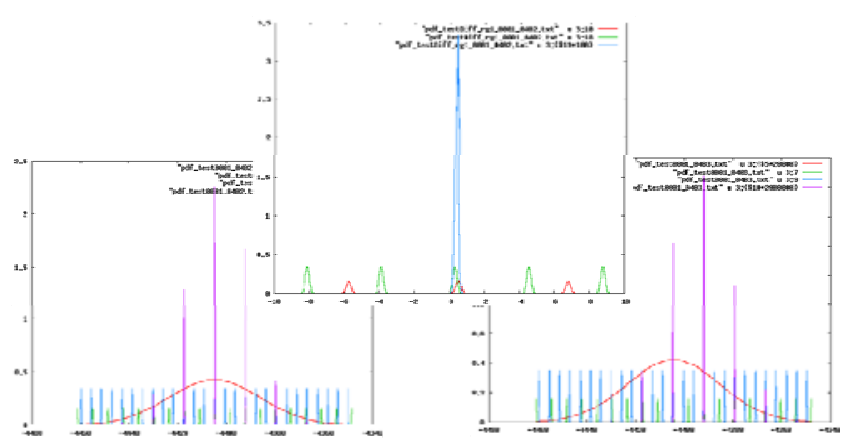

Figure 2: Bottom right and left, pdf of the neighboring pixels, one with wrong maximum; top, maximum likelihood estimator for their gradient which gives the correct peak

\subsection{Delta-K Absolute Phase Estimation}

Estimation of the absolute interferometric phase without error prone phase unwrapping (PU) is possible with TerraSAR-X by exploiting the frequency diversity within the up to $300 \mathrm{MHz}$ wide bandwidth signal to implicitly determine the phase ambiguity. One such technique, the delta-k method first proposed by Madsen [9], is currently being studied at the DLR within the framework of an ESA study $(21318 / 07 / \mathrm{NL} / \mathrm{HE})$. It is envisaged as an independent means for supporting and/or validating the multibaseline PU to be used for the TanDEM-X mission.

The optimal estimator of shift or phase between two SAR images is the complex cross correlation [10]. Delta-k offers a way of performing the complex correlation in a slightly suboptimal but computationally efficient way. The SAR images are first bandpass filtered into non-overlapping lower and upper subbands from which lower and upper subband interferograms are formed. The so called delta-k interferogram - a differential interferogram between the two 
subband interferograms - directly provides an estimate of the phase-frequency gradient. The relationship between the delta-k phase, $\Delta \phi$, taken from the delta-k interferogram and the differential range, $\Delta \mathrm{r}$, is, $\Delta \phi=4 \pi \frac{\mathrm{f}_{\Delta}}{\mathrm{c}} \Delta \mathrm{r}$, where $\mathrm{f}_{\Delta}=\mathrm{B} / \mathrm{b}$ is the delta-k frequency, B the range bandwidth of the SAR system, $b$ the subband bandwidth and $c$ the speed of light. Comparing this to the corresponding relation for standard repeat-pass interferometry, $\phi=4 \pi \frac{\mathrm{f}_{\mathrm{C}}}{\mathrm{c}} \Delta \mathrm{r}$, where $\phi$ is the interferometric phase and $\mathrm{f}_{\mathrm{C}}$ the radar carrier frequency, shows that delta-k simulates an interferogram from a SAR system with carrier frequency $f_{\Delta}$. Choosing $b=B / 3$ optimally trades off estimator variance and resolution [10], giving $\mathrm{f}_{\Delta}=2 \mathrm{~B} / 3$. This increases the height of ambiguity (HoA) by a factor $\mathrm{f}_{\mathrm{C}} / \mathrm{f}_{\Delta}=9.65 \mathrm{GHz} / 200 \mathrm{MHz} \approx 50$, at which point $\mathrm{PU}$ becomes unnecessary. To avoid phase wraps implies a phase error less than \pm 0.5 cycles at $f_{C}$. The phase variance at $\mathrm{f}_{\Delta}$ must therefore be reduced by a factor of approximately $\left(\mathrm{f}_{\mathrm{C}} / \mathrm{f}_{\Delta}\right)^{2}$ to avoid phase wraps at $\mathrm{f}_{\mathrm{C}}$, this is achieved by smoothing the delta-k phase. Hence, delta-k provides an absolute phase estimate at a lower resolution. Further details on the processing can be found in [11].

Figure 3 shows an example of absolute phase estimation using conventional MCF PU and the delta-k estimator for the salt lake Salar de Arizaro, Argentina. Acquisitions were made with the TSX-1 satellite in stripmap mode with a range bandwidth of $100 \mathrm{MHz}$. The standard interferogram has a resolution of $4.5 \times 3 \mathrm{~m}$ and a HoA of $7.4 \mathrm{~m} /$ cycle.

For delta-k processing the optimal subband bandwidth of $\mathrm{b}=33 \mathrm{MHz}$ gives a HoA of $1076 \mathrm{~m} /$ cycle and a resolution of $190 \times 300 \mathrm{~m}$. The change in topography of about $1500 \mathrm{~m}$ is then greater than the delta-k HoA, still requiring PU. However, MCF PU for delta-k was trivial with zero residues, i.e. no ambiguities. Visually, the delta-k absolute phase fringe pattern matches well to the MCF PU estimate the reduced resolution of delta-k due to smoothing being the major difference. A detailed analysis where the delta-k and MCF-PU estimates were compared both to each other and to an SRTM DEM showed that MCF-PU resulted in many more PU errors of a much larger magnitude in comparison to the delta-k absolute phase estimate.
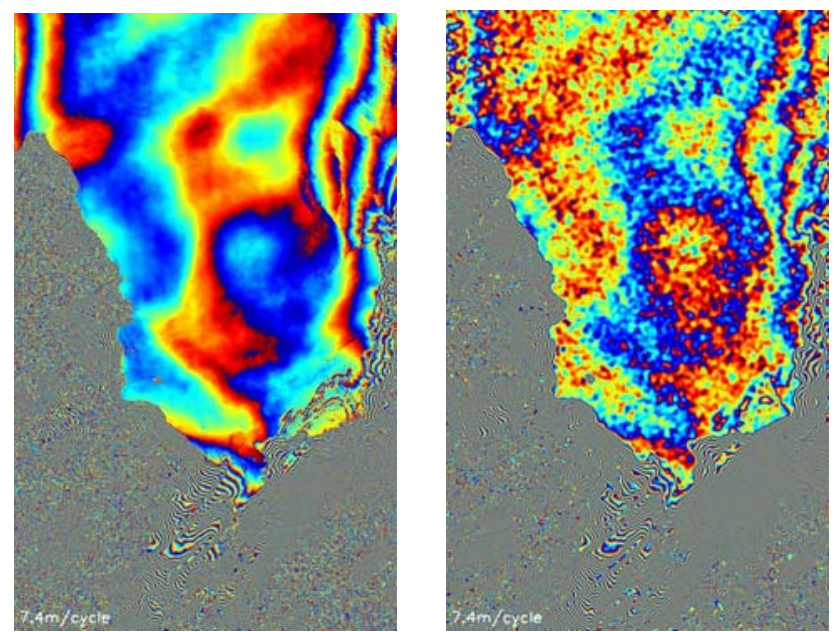

Figure 3: Rewrapped absolute phase estimate from (left) MCF PU of the standard interferogram and (right) delta-k. In the images, bottom left corresponds to near range, early azimuth and upper right to far range, late azimuth

\section{REFERENCES}

[1] G. Krieger, A. Moreira, H. Fiedler, I. Hajnsek, M. Werner, M. Younis and M. Zink, "The TanDEM-X mission: a satellite formation for high resolution SAR interferometry," IEEE Transactions on Remote Sensing, 2007, in press.

[2] N. Adam, M. Eineder, B. Schättler, and N. Yague-Martinez, "First TerraSAR-X Interferometry Evaluation," Proceedings of ESA FRINGE Workshop, Frascati, 2007.

[3] M. Eineder, "Ocillator clock drift compensation in bistatic interferometric SAR," Vol. 3, pp. 1449 - 1451, 2003.

[4] R. Bamler, F. Meyer, W. Liebhart, "Processing of Bistatic SAR Data From Quasi-Stationary Configurations," IEEE Transactions on Geoscience and Remote Sensing, Vol. 45, Issue 11, Part 1, pp. 3350 - 3358, 2007.

[5] Krieger, G., Younis, M., "Impact of Oscillator Noise in Bistatic and Multistatic SAR," IEEE GRSL, Vol.3, July 2006

[6] S. Suchandt and M. Eineder, "Experiences with SRTM / XSAR Phase Unwrapping using the Minimum Cost Flow Method," Proc. of IEEE International Geoscience and Remote Sensing Symposium, Toulouse, 2003.

[7] M. Eineder, N. Adam, "A maximum likelihood estimator to simultaneously unwrap, geocode and fuse SAR interferograms from different viewing geometries into one digital elevation model," IEEE Transactions on Geoscience and Remote Sensing, Vol. 43, Iss. 1, pp. 24-36, 2005.

[8] M. Eineder, "Interferometric DEM Reconstruction of Alpine Areas - Experiences With SRTM Data and Improved Strategies for Future Missions," Proceedings of EARSEL 3D workhop, Porto, 2005.

[9] S. N. Madsen, "On absolute phase determination techniques in SAR interferometry," Proceedings SPIE Conference on Radar Sensor Technology, vol. 2487, pp. 393-401, April 1995.

[10] R. Bamler and M. Eineder, "Accuracy of differential shift estimation by correlation and split-bandwidth interferometry for wideband and delta-k SAR systems," IEEE Geoscience and Remote Sensing Letters, vol. 2, no. 2, pp. 151-155, April 2005.

[11] R. Brcic, M. Eineder and R. Bamler, "Interferometric Absolute Phase Determination with TerraSAR-X Wideband SAR Data," Proceedings RadarCon09, May 2009. 